\title{
EL CONSUMO DE PAPEL Y SUS IMPLICACIONES SOBRE LOS BOSQUES Y EL MEDIOAMBIENTE
}

\section{Miguel Ángel Soto Caba}

\author{
GREENPEACE ESPAÑA \\ masoto@es.greenpeace.org
}

Si nos atenemos a los datos oficiales sobre la procedencia del papel que se consume en España y echamos un vistazo rápido a los papeles utilizados en los medios de expresión escrita de una parte del movimiento ecologista, las revistas de divulgación de la naturaleza, las editoriales afines o la misma administración ambiental, caeremos en la cuenta de que los defensores del medio ambiente, utilizamos en nuestros medios de expresión productos de papelería que proceden de la destrucción de los bosques primarios de Finlandia y Canadá, de la devastación de las selvas de Indonesia, o de los polémicos y mal gestionados cultivos de eucaliptos en la península ibérica. No es esta la única contradicción que encontramos sobre los hábitos de consumo de papel en nuestro país. El sector papelero y las empresas de distribución de papel han conseguido trasmitir diversas falsedades que han llegado a calar fuerte en el mercado de papel, a saber: el papel reciclado contamina más que el blanco; el papel reciclado estropea las fotocopiadores e impresoras; el papel reciclado es más caro; y, por último, el "papel ecológico" verdadero es el papel de fibra virgen libre de cloro elemental (ECF), precisamente el que fabrica mayoritariamente el sector papelero español.
El resultado de esta información confusa procedente del sector papelero local, se ha traducido en un descenso de las ventas de papel reciclado en España. Administraciones públicas que a mediados de los años 90 tenían una política de compras de papel reciclado para el consumo interno, han dado pasos atrás y consumen hoy en día papel blanco de fibra virgen. Hasta el Ministerio de Medio Ambiente y la Dirección General de Conservación de la Naturaleza han sucumbido al empuje.

\section{INDICADOR DE DERROCHE}

Pero la premisa más peligrosa defendida desde el sector pastero y papelero es que el crecimiento de la demanda de papel es inevitable. El aumento del consumo de papel es utilizado por los analistas del sector como señal de crecimiento económico saludable y de mejora de la calidad de vida, como un "indicador de desarrollo".

Pero el aumento del consumo del papel en el mundo y las previsiones de crecimiento que se hacen de él se sustentan en modelos económicos insostenibles, enormemente derrochadores y contrarios al principio de precaución con el Medio Ambiente. En realidad, el aumento de la demanda 
de papel es un indicador de despilfarro de los recursos naturales. Las naciones industrializadas, con el $20 \%$ de la población mundial, consumen el $87 \%$ del papel para escribir e imprimir ${ }^{1}$. Consumen pero, sobre todo, derrochan recursos, ya que entre el 30 y el $40 \%$ de los residuos sólidos urbanos generados en Europa son papel y cartón ${ }^{2}$. Usamos demasiado papel y, además, no lo reciclamos. El consumo de papel en España era, en 2002, de algo más de $170 \mathrm{~kg} / \mathrm{habitante/año,} \mathrm{alejados} \mathrm{todavía} \mathrm{de}$ los $206 \mathrm{~kg} / \mathrm{habitante}$ de Reino Unido o los 225

1 Klaus Toepfer, Director Ejecutivo, Programa Medioambiental de la ONU, (...) VII Seminario Internacional sobre Producción Más Limpia, 29-30 Abril 2002.

2 WorldWatch Institute, 2000. Signos Vitales 2000. Bakeaz y Gaia Proyecto 2050. $\mathrm{kg} / \mathrm{habitante}$ de Alemania ${ }^{3}$. Este dato sirve al sector papelero para reclamar y predecir un mayor consumo de papel para acercarnos a los países "más avanzados".

Pero los españoles desperdiciamos un millón de toneladas de envases de papel y cartón y 850.000 toneladas de papeles de impresión y escritura ${ }^{4}$, un $40 \%$ del papel y cartón consumido. Además, pese al importante incremento de la recuperación de papel en nuestro país, sigue siendo insuficiente para cubrir la capacidad de reciclaje de la industria papelera española. Este sector se ve obligado a importar anualmente del orden del 14\% del consumo de papel recuperado de otros países.

3 Aspapel, 2003. Informe Estadístico 2002.

4 Aspapel, 2003. Diagnóstico de la Recuperación de Papel y Cartón en España.

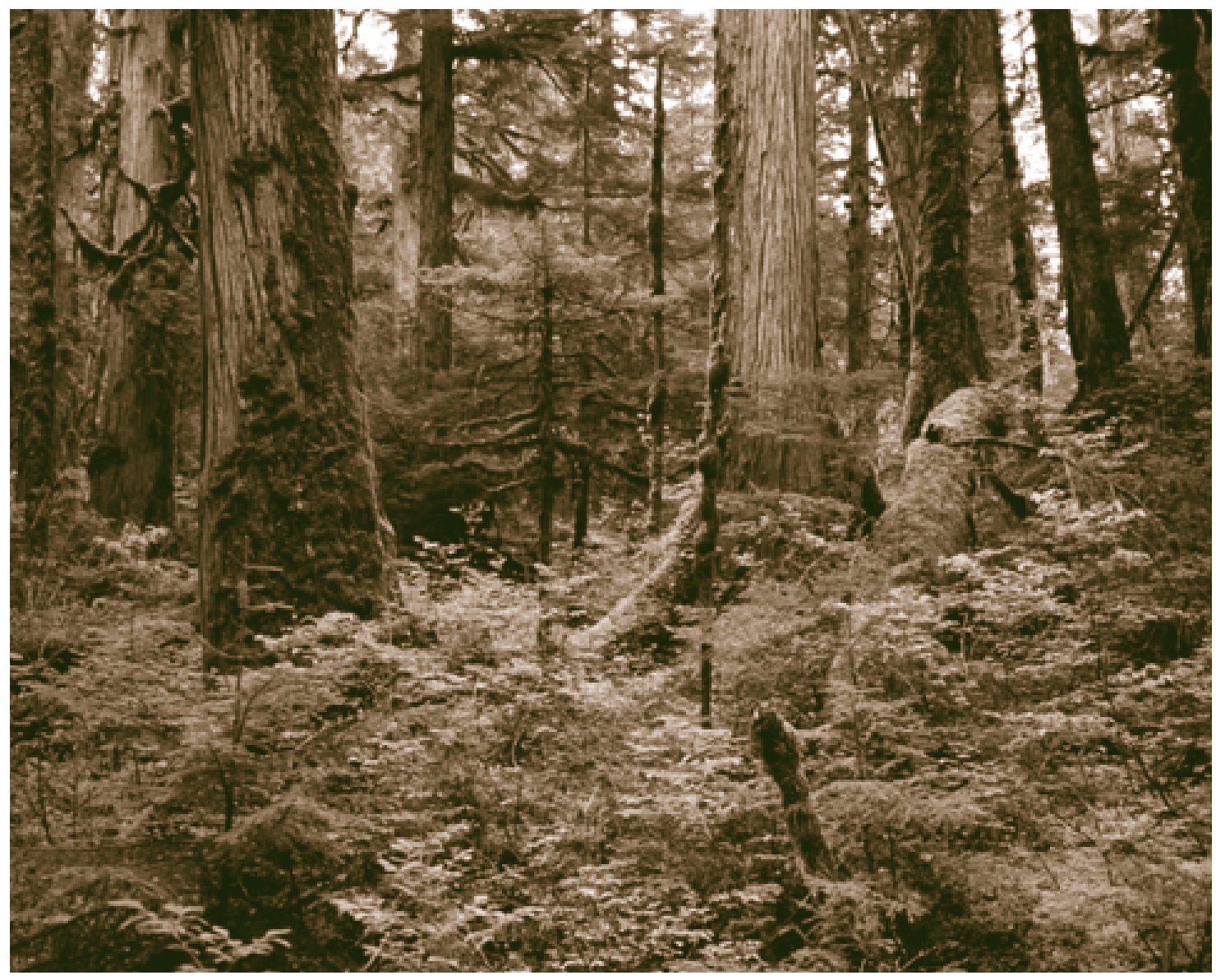

Figura 1. Bosque primario Canadiense. 
Pero el incremento de utilización del papel recuperado no ha supuesto un alivio para las masas forestales españolas ni para los Bosques Primarios (a través de las importaciones de productos papeleros). Dentro de nuestras fronteras, y pese al fuerte avance en el consumo de papel usado, la cantidad de madera cortada en los montes españoles no ha disminuido5. El efecto ha sido que la demanda de papel recuperado por parte de la industria cartonera, ha servido para fomentar la importación de papel viejo extraído de otros países, y no para mejorar la recogida de nuestro papel usado o fomentar el consumo de papel reciclado. De hecho, los fabricantes españoles de papel reciclado se quejan del descenso en la demanda de papel de escritura reciclado.

\section{UN PROBLEMA URGENTE}

La industria papelera y de celulosa ocupa el quinto lugar del sector industrial en consumo mundial de energía, y utiliza más agua por cada tonelada producida que cualquier otra industria. También, la industria pastero-papelera se encuentra entre los mayores generadores de contaminantes del aire y del agua, así como gases que causan el cambio climático ${ }^{6}$.

La fabricación y consumo de papel y el futuro de los bosques están estrechamente unidos, aunque frecuentemente la cantidad de madera empleada en la fabricación de pastas vírgenes se infravalora al no contabilizarse los consumos de restos y residuos de aserraderos. Lo cierto es que cerca del $40 \%$ de toda la madera talada para usos industriales en el mundo se destina a la producción de papel ${ }^{7}$ : el $25 \%$ son cortas directas para la industria del papel, mientras que el otro $15 \%$ restante procede de subproductos de otros sectores (aserrío, fabricación de tableros, etc.).

Las fuentes de esas fibras, según los tipos de masas forestales son las siguientes: $17 \%$ procede de

5 StoraEnso, 1999. 144 Cuestiones sobre el papel y el cartón. Fundación Terra.

6 WorldWatch Institute, 2004. La situación del mundo 2004 Icaria Editorial y Fuhem.

7 WorldWatch Institute, 2000. La situación del mundo 2000. Icaria Editorial y Fundación Hogar del Empleado.
Bosques Primarios (bosques vírgenes), sobre todo en regiones boreales; el $54 \%$ de bosques secundarios; y, por último, el 29\% de plantaciones forestales ${ }^{8}$.

En muchos lugares del planeta, los bosques y otros ecosistemas naturales han sido y son todavía sustituidos por plantaciones de árboles de crecimiento rápido cuya gestión implica la utilización masiva de herbicidas y fertilizantes químicos tóxicos. Además, los monocultivos de árboles para la industria del papel son frecuentemente rechazados por las comunidades locales debido a sus impactos negativos sobre los modos de vida y el bienestar de la población?.

\section{LA PRODUCCIÓN Y CONSUMO DE PAPEL EN ESPAÑA}

El sector pastero-papelero español consumió en 2003 un total de $5.858 .000 \mathrm{~m}^{3}$ de madera como materia prima para la fabricación de 1.894 .000 toneladas de celulosa, lo que supuso un crecimiento del $8,9 \%$ con respecto al año anterior. La madera utilizada para la fabricación de celulosa en España procede de plantaciones de especies de crecimiento rápido: 4,7 millones $\mathrm{de}^{3}$ de Eucaliptus globulus y 1,2 millones de $\mathrm{m}^{3}$ de resinosas, principalmente Pinus radiata.

La madera procedente de la importación supuso el $23 \%$ del consumo tota ${ }^{10}$. Con esta materia prima, una buena parte de las fábricas españolas producen pasta química blanqueada al sulfato, un proceso de producción anticuado que genera muchos residuos y con un impacto muy negativo sobre el Medio Ambiente.

Una parte importante de la industria papelera española, la que se dedica a la fabricación de cartón para embalaje, recicla al año cerca de 4 millones de toneladas de papel recuperado, evitando que acabe en los vertederos. De este modo, el sector papelero emplea como materia prima una media de 8 tone-

8 World Watch Institute, 2000. Signos Vitales 2000. Bakeaz y Gaia Proyecto 2050.

9 Movimiento Mundial por los Bosques. Boletín no 83, junio de 2004. Los impactos de la producción de celulosa. http://www.wrm.org.uy/inicio.html

10 Aspapel, 2004. Comunicado de Prensa, 26 de abril de 2004. 
ladas de papel recuperado por cada 10 toneladas de papel nuevo que se fabrica. Son los papeles de envases y embalajes los que utilizan un mayor aporte de fibra reciclada. La mayor inyección de fibra virgen en el proceso se produce a través de los papeles de impresión y escritura.

El sector papelero español todavía utiliza el nocivo cloro en el proceso de blanqueo de la pasta, bien sea como cloro gaseoso (cloro elemental) o dióxido de cloro (ECF, libre de cloro elemental). Así, los compuestos organoclorados generados durante el proceso, entre los que se encuentran las temidas dioxinas, siguen comprometiendo la salud de los ciudadanos y la del planeta. Sólo una pequeña parte de la producción es totalmente libre de cloro (TCF).

\section{COMPRANDO DESTRUCCIÓN FUERA DE NUESTRAS FRONTERAS}

Dada la especialización del sector español en la producción de pasta de celulosa y embalajes de cartón, la demanda interna de papel de oficina y de impresión, así como el pujante sector editorial español, obliga a realizar importaciones de productos papeleros desde regiones con recursos forestales. Los datos oficiales sobre importación de pasta papelera y papel para impresión demuestran que el mercado español está contribuyendo a la destrucción de los Bosques Primarios del mundo $^{11}$ mediante una política de aprovisionamiento con pocos criterios sociales y ambientales, cuando no inexistentes. Recordemos que el $17 \%$ de la madera talada industrialmente en el mundo procede de estos bosques de enorme valor ecológico y cultural.

El principal país proveedor de productos de papel y cartón es Finlandia, con 608.644 mil toneladas en 2001. Los Bosques Primarios finlandeses están siendo destruidos o transformados para la

11 Bosques Primarios (conocidos también como Ancient Forest, Old Growth Forest, Bosque Nativo) son superficies continuas de bosque original intacto, cuya dinámica y evolución depende del régimen de perturbaciones naturales, con nula o escasa intervención humana, y lo suficientemente grandes para garantizar la supervivencia de poblaciones viables de todos los seres vivos, incluidas especies migratorias. fabricación de papel. En el año 2002 se importaron 157.135 toneladas de papel de impresión. Tres empresas producen la mayoría de este papel: StoraEnso, M-Real y UPM-Kymmene. También en Canadá los Bosques Primarios están siendo destruidos por la industria de la celulosa y el papel. En el año 2002 se importaron de este país 82.079 toneladas de pastas papeleras y 60.266 toneladas de papel y cartón.

El Sureste Asiático, paraíso de la tala ilegal, también provee de productos papeleros al mercado español ${ }^{12}$. En países como Indonesia, donde las plantaciones se cuadriplicaron en la década de los 90, más de 1,4 millones de hectáreas de Bosque Primario intacto han sido reemplazadas por cultivos forestales, fundamentalmente de eucaliptos y otras especies utilizadas por el sector papelero ${ }^{13}$, obligando a las poblaciones indígenas que los habi$\tan$ a dejar sus tierras ${ }^{14}$. En el año 2002, el sector papelero y editorial español importó de esta región 8.710 toneladas de pastas papeleras y 28.180 toneladas de papel, mayormente de papel para impresión y escritura.

\section{EL CURRICULUM AMBIENTAL DEL SEC- TOR PAPELERO ESPAÑOL}

Los procesos tecnológicos de producción de pasta y papel han mejorado en las últimas décadas y, aunque todavía se producen episodios de agresión al Medio Ambiente, hay que reconocer que el sector se ha visto obligado a acometer mejoras tecnológicas, implantar sistemas de gestión ambiental y reducir su impacto sobre el Medio Ambiente.

Pero lo cierto es que el sector pastero-papelero español se ha significado históricamente por su poca preocupación por el cuidado del Medio Ambiente y la salud de los ciudadanos. Además de contaminar en el pasado gravemente ríos y zonas

12 Se estima que el $90 \%$ de las cortas industriales de madera en Indonesia son ilegales.

13 WorldWatch Institute, 2000. La situación del mundo 2000. Icaria Editorial y Fundación Hogar del Empleado.

14 Según un informe de 1995 de WWF "Indonesia es quizás el único país donde la expansión planificada de la industria papelera implica abiertamente la deforestación de áreas importantes de bosques tropicales”. Citado por Ashley T. Matoon en la revista de WorldWatch 1998 
costeras, su influencia y poder en el sector forestal ha generado que España tenga la mayor superficie ocupada por Eucalyptus globulus del mundo (fuera de su área natural), pese a ser una especie nativa de Australia. España es un claro ejemplo del problema de las plantaciones de eucalipto y otras especies utilizadas por el sector papelero, como el Pinus radiata.

El esfuerzo propagandista y económico del "lobby" del eucalipto durante décadas ha buscado la identificación de los cultivos forestales de celulosa con la alternativa única y viable, y la identificación de la política celulósica con la política forestal, provocando la ruptura abierta con más de un siglo de tradición y técnica forestal en España. Pero los problemas ambientales y sociales de la política de "eucaliptización" del paisaje ibérico han sido contestados desde la sociedad civil y la comunidad científica, que desde la década de los 80 han clamado contra el monopolio forestal del sector papelero advirtiendo de sus peligros y falta de visión a largo plazo. Las consecuencias de la plantación masiva de eucaliptos en zonas como Galicia, Asturias, Cantabria o Huelva han sido la destrucción de bosque y matorral autóctono, la agresión continua a la biodiversidad de nuestros ecosistemas y la trivialización de nuestros paisajes hasta convertirlos en fábricas de celulosa.

La gestión forestal de estas plantaciones no es precisamente sostenible. Para Greenpeace y otras organizaciones ecologistas, sociales y sindicales, el Forest Stewardship Council (FSC) es el esquema de certificación forestal internacional más creíble y que se acerca mejor a este objetivo.

No contentos con la situación creada, la patronal del sector, ASPAPEL ha seguido en los últimos años reclamando a la administración que se dediquen 200.000 hectáreas más para plantaciones de especies de crecimiento rápido para la industria papelera, pese a que esta superficie sigue cre-

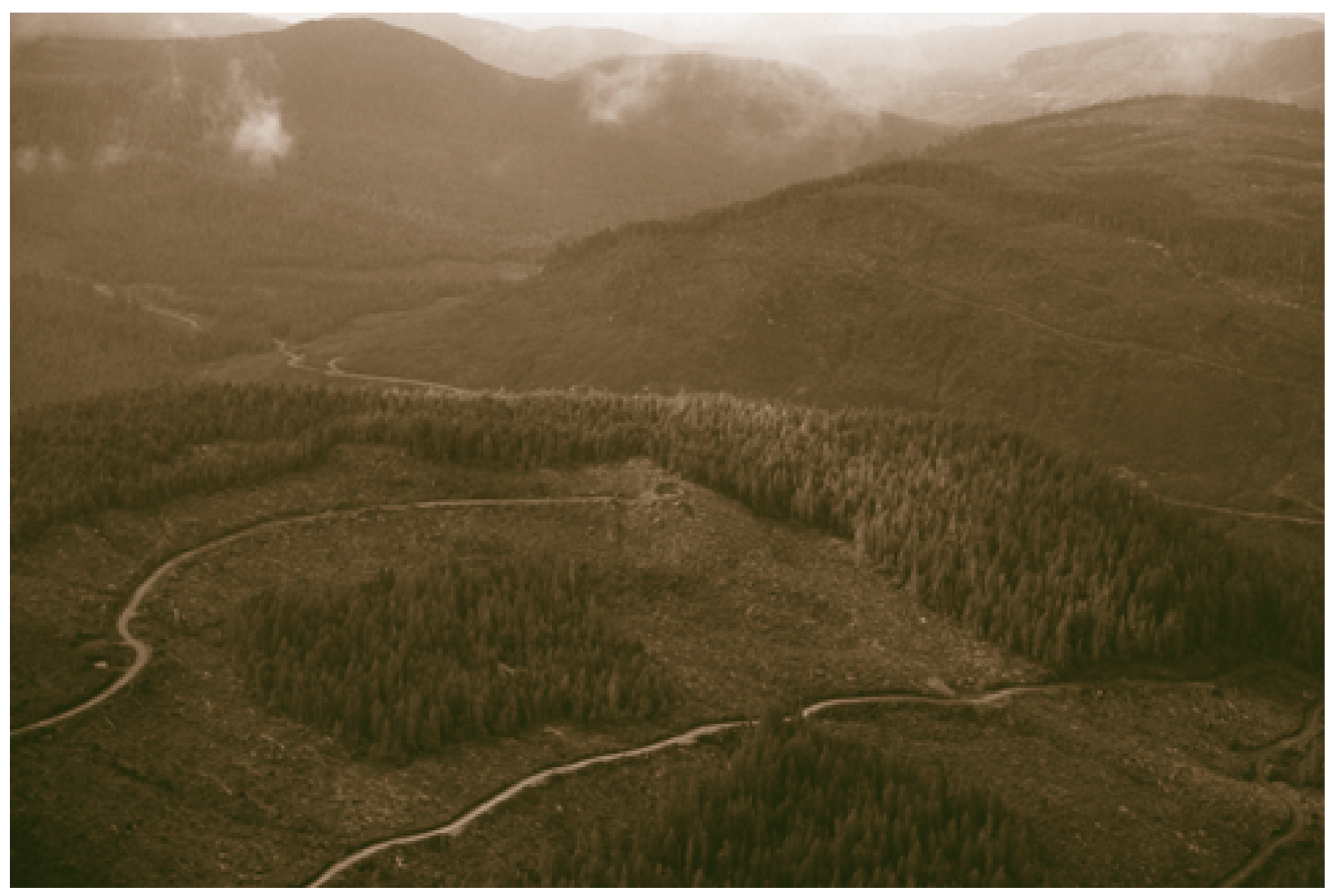

Figura 2. Deforestación de un bosque de coniferas. 
ciendo año a año ${ }^{15}$. ASPAPEL ha sido también promotora de sistemas de certificación forestal que no mejoran la gestión forestal, no son transparentes y no dan oportunidad a la participación real de la sociedad civil.

El último gesto del sector papelero español se produjo en abril de 2004, cuando el presidente de ASPAPEL se sumó al resto del sector empresarial opuesto al Protocolo de Kioto, declarando que dicho acuerdo multilateral no se puede alcanzar $^{16}$.

\section{El papel "Amigo de los bosques"}

Dadas las implicaciones ambientales del consumo de papel, a la hora de elegir los productos papeleros debemos tener en cuenta tanto el origen de la fibra de celulosa, como los procesos de producción y blanqueo. Así, son preferibles:

15 Montoya Oliver, experto forestal reconocido en nuestro país, afirma en su libro El Eucalipto (1995, Editorial Mundi-Prensa) que "es conveniente no plantar en España una extensión excesiva de eucaliptos, pues podría correrse el riesgo de que la oferta superara la demanda en algunos casos".

16 La Gaceta de los Negocios, 23/04/2004. La patronal del papel dice que Kioto es inviable.
- Como primera opción y para la mayoría de los usos, el papel $100 \%$ reciclado post-consumo. La etiqueta ecológica "Angel Azul" ayuda a identificar este tipo de papel. Otras opciones con alto contenido en fibra reciclada también son bienvenidas.

- Cuando es imprescindible el papel blanco, de fibra vírgen, hay que asegurarse que no proceda de empresas y/o países donde se están destruyendo bosques de Alto Valor para la Conservación, incluidos bosques primarios. Para tener garantías de la sostenibilidad de la gestión forestal el papel deberá estar certificado por el Forest Stewardship Council, más conocido por las siglas FSC.

- Existen en el mercado papeles de oficina fabricados a partir de fibras agrícolas (bagazo de caña de azúcar por ejemplo) y/o cultivos no madereros. Esto disminuye la presión sobre los bosques y la proliferación de plantaciones.

- El blanqueado deber ser totalmente libre de cloro. Se utiliza el término procesado totalmente libre de cloro (PCF) para el caso de la fibra reciclada, o totalmente libre de cloro (TCF) para la fibra virgen. 